3. R. B. Crittenden and V. L. Shapiro, Sets of uniqueness on the group $2^{\omega}$, Ann. of Math. (2) 81 (1965), 550-564. MR 31 \#3783.

4. W. R. Wade, Uniqueness theory for Cesaro summable Haar series, Duke Math. J. 38 (1971), 221-227.

5. Uniqueness of Haar series which are $(C, 1)$ summable to Denjoy integrable functions, Trans. Amer. Math. Soc. 176 (1973), 489-498. MR 47 \#704.

6. R. J. Lindahl, A differentiation theorem for functions defined on the dyadic rationals, Proc. Amer. Math. Soc. 30 (1971), 349-352. MR $44 \# 1774$.

DEPARTMENT OF MATHEMATICS, UNIVERSITY OF TENNESSEE, KNOXVILLE, TENNESSEE 37916

PROCEEDINGS OF THE

AME RICAN MATHEMATICAL SOCIETY

Volume 50, July 1975

\title{
$L_{p, q}$ MODULARS $^{*}$
}

\section{HIDEGORO NAKANO}

ABSTRACT. For $1<p \leq q$, a convex modular $m$ on a linear space $S$ is called an $L_{p, q}$ modular if $\operatorname{Min}_{r=p, q} \xi^{r} m(x) \leq m(\xi x) \leq \operatorname{Max}_{r=p, q} \xi^{r} m(x)$ for $\xi>0$ and $x \in S$. We generalize the Minkowski inequality and the Hölder inequality for $L_{p, q}$ modulars.

For $1<p \leq q$, a convex modular $m$ on a linear space $S$ is called an $L_{p, q}$ modular if

and

$$
\xi^{p} m(x) \leq m(\xi x) \leq \xi^{q} m(x) \text { for } \xi \geq 1 \text { and } x \in S,
$$

$$
\xi^{p} m(x) \geq m(\xi x) \geq \xi^{q} m(x) \text { for } 0<\xi \leq 1 \text { and } x \in S .
$$

An $L_{p, q}$ modular is $p$-homogeneous if $p=q$, as defined in [3]. We generalized the Minkowski inequality and the Hölder inequality for $p$-homogeneous modu-

Received by the editors July 11, 1972 and, in revised form, September 27, 1973. AMS (MOS) subject classifications (1970). Primary 46B99.

Key words and phrases. Normed linear spaces, Banach spaces.

* This paper is being published posthumously. Galley proofs were corrected by Professor Kazumi Nakano, Department of Mathematics, SUNY at Brockport, Brockport, New York 14420. 
lars in [3] by the theory of modular spaces in [1] and [2]. In this paper, we will generalize them for $L_{p, q}$ modulars.

For the second modular norm $N_{2}$ of $m$, we have

$$
N_{2}(x)=\inf _{m(\xi x) \leq 1, \xi>0} \frac{1}{\xi} \text { for } x \in S,
$$

and we have $N_{2}(x) \leq 1$ if and only if $m(x) \leq 1$, as proved in [1] and [2]。 If $m(x) \leq 1$, then it is clear that $N_{2}(x)=\inf _{m(\xi x) \leq 1, \xi \geq 1}(1 / \xi)$. Since $\xi^{p} m(x) \leq$ $m(\xi x) \leq \xi^{q} m(x)$ for $\xi \geq 1$, we obtain

$m(x)^{1 / p}=\inf _{\xi^{p} m(x) \leq 1, \xi \geq 1} \frac{1}{\xi} \leq N_{2}(x) \leq \inf _{\xi^{q} m(x) \leq 1, \xi \geq 1} \frac{1}{\xi}=m(x)^{1 / q}$, if $m(x) \leq 1$.

That is, $m(x)^{1 / p} \leq N_{2}(x) \leq m(x)^{1 / q}$ if $m(x) \leq 1$. Likewise, we obtain $m(x)^{1 / q} \leq N_{2}(x) \leq m(x)^{1 / p}$ if $m(x) \geq 1$. Therefore we have

$$
\operatorname{Min}_{r=p, q} m(x)^{1 / r} \leq N_{2}(x) \leq \operatorname{Max}_{r=p, q} m(x)^{1 / r} \text { for } x \in S .
$$

Since $N_{2}(x+y) \leq N_{2}(x)+N_{2}(y)$, we obtain a generalization of the Minkowski inequality

$$
\operatorname{Min}_{r=p, q} m(x+y)^{1 / r} \leq \operatorname{Max}_{r=p, q} m(x)^{1 / r}+\operatorname{Max}_{r=p, q} m(y)^{1 / r} .
$$

For the first modular norm $N_{1}$ of $m$, we have

$$
N_{1}(x)=\inf _{\xi>0} \frac{1}{\xi}(1+m(\xi x)) \text { for } x \in S,
$$

as proved in [1] and [2]. If $N_{1}(x)=\inf _{1 \geq \xi>0}(1 / \xi)(1+m(\xi x))$, then

$$
\begin{aligned}
q^{1 / q}{q^{\prime}}^{1 / q^{\prime}} m(x)^{1 / q} & =\inf _{\xi>0} \frac{1}{\xi}\left(1+\xi^{q} m(x)\right) \leq N_{1}(x) \\
& \leq \inf _{\xi>0} \frac{1}{\xi}\left(1+\xi^{p} m(x)\right)=p^{1 / p}{p^{\prime}}^{1 / p^{\prime}} m(x)^{1 / p}
\end{aligned}
$$

for $1 / p+1 / p^{\prime}=1$ and $1 / q+1 / q^{\prime}=1$. If $N_{1}(x)=\inf _{\xi \geq 1}(1 / \xi)(1+m(\xi x))$, then

$$
p^{1 / p}{p^{\prime}}^{1 / p^{\prime}} m(x)^{1 / p} \leq N_{1}(x) \leq q^{1 / q} q^{1^{1 / q^{\prime}}} m(x)^{1 / q} .
$$

Therefore

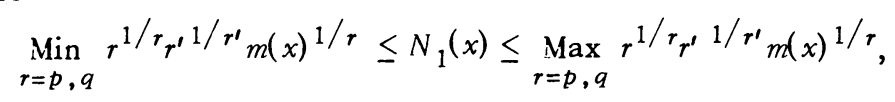

for $1 / p+1 / p^{\prime}=1,1 / q+1 / q^{\prime}=1$, and $x \in S$.

Let $\bar{S}$ be the conjugate space of $S$ and let $\bar{m}$ be the conjugate modular of an $L_{p, q}$ modular $m: \bar{m}(\phi)=\sup _{x \in S}\{\phi(x)-m(x)\}$ for every $\phi \in \bar{S}$. By definition, 


$$
\bar{m}(\xi \phi)=\sup _{x \in S}\{\phi(\xi x)-m(x)\}=\sup _{x \in S}\{\phi(x)-m(x / \xi)\}
$$

for every $\xi \neq 0$. In case $\xi \geq 1, m(x / \xi) \geq \xi^{\alpha p} m\left(x / \xi^{1+\alpha}\right)$ if $\alpha=1 /(p-1)$. Then,

$$
\bar{m}(\xi \phi) \leq \sup _{x \in S}\left\{\phi(x)-\xi^{a p} m\left(x / \xi^{1+a}\right)\right\}=\xi^{a p} \sup _{x \in S}\left\{\phi\left(x / \xi^{\alpha p}\right)-m\left(x / \xi^{1+a}\right)\right\} .
$$

Let $1 / p+1 / p^{\prime}=1$. Then $p /(p-1)=1+1 /(p-1)=p^{\prime}$ and by replacing $\alpha p$ and $\alpha+1$ in the above inequality, with $p^{\prime}$,

$$
\bar{m}(\xi \phi) \leq \xi^{\prime \prime} \sup _{x \in S}\{\phi(x)-m(x)\}=\xi^{p^{\prime}} \bar{m}(\phi) .
$$

In a similar manner, we can prove that $\bar{m}(\xi \phi) \geq \xi^{q^{\prime}} \bar{m}(\phi)$ for $q^{\prime}$ such that $1 / q+1 / q^{\prime}=1$. Therefore, if $m$ is an $L_{p, q} \operatorname{modular}$, and $1 / p+1 / p^{\prime}=1$ and $1 / q+1 / q^{\prime}=1$ then $\xi^{q^{\prime}} \bar{m}(\phi) \leq \bar{m}(\xi \phi) \leq \xi^{p^{\prime}} \bar{m}(\phi)$ for every $\xi \geq 1$ and every $\phi \in \bar{S}$. A similar computation also leads us to the following inequality, in case $0<\xi \leq 1$ :

$$
\xi^{p^{\prime}} \bar{m}(\phi) \leq \bar{m}(\xi \phi) \leq \xi^{q^{\prime}} \bar{m}(\phi) \quad \text { for every } \phi \in \bar{S} \text {. }
$$

Hence the conjugate modular $\bar{m}$ of an $L_{p, q}$ modular $m$, is an $L_{q^{\prime}, p^{\prime}}$ modular

For the first and second modular norms $\bar{N}_{1}$ and $\bar{N}_{2}$ of $\bar{m}$, we have

$$
|\phi(x)| \leq \bar{N}_{1}(\phi) N_{2}(x) \text { and }|\phi(x)| \leq \bar{N}_{2}(\phi) N_{1}(x)
$$

for $\phi \in \bar{S}$ and $x \in S$. Therefore we can generalize the Hölder inequality as

$$
|\phi(x)| \leq \operatorname{Max}_{r=p, q} r^{1 / r^{\prime} r^{1 / r^{\prime}}} \bar{m}(\phi)^{1 / r^{\prime}} \operatorname{Max}_{r=p, q} m(x)^{1 / r},
$$

and

$$
|\phi(x)| \leq \operatorname{Max}_{r=p, q} \bar{m}(\phi)^{1 / r^{\prime}} \operatorname{Max}_{r=p, q} r^{1 / r} r^{1 / r^{\prime}} m(x)^{1 / r} .
$$

As an example, we consider the linear space $S_{0}$ of all continuous functions on the closed interval $[0,1]$. For $\rho \in S_{0}$ with $1<p \leq \rho(t) \leq q$ for $t \in[0,1]$, setting

$$
m(\phi)=\int_{0}^{1}|\phi(t)|^{\rho(t)} d t \quad \text { for } \phi \in S_{0},
$$

we obtain an $L_{p, q}$ modular $m$ on $S_{0^{\circ}}$. By (1) we have a generalization of the Minkowski inequality

$$
\begin{aligned}
& \operatorname{Min}_{r=p, q}\left(\int_{0}^{1}|\phi(t)+\psi(t)|^{\rho(t)} d t\right)^{1 / r} \\
& \quad \leq \operatorname{Max}_{r=p, q}\left(\int_{0}^{1}|\phi(t)|^{\rho(t)} d t\right)^{1 / r}+\operatorname{Max}_{r=p, q}\left(\int_{0}^{1}|\psi(t)|^{\rho(t)} d t\right)^{1 / r}
\end{aligned}
$$

if $1<p \leq \rho(t) \leq q$ for $t \in[0,1]$. 
We also set

$$
m_{0}(\phi)=\int_{0}^{1} \frac{1}{\rho(t)}|\phi(t)|^{\rho(t)} d t \quad \text { for } \phi \in S_{0} .
$$

Then $m_{0}$ is an $L_{p, q}$ modular on $S_{0^{*}}$. For the conjugate space $\bar{S}_{0}$ of $S_{0}$ for $m_{0}$, we can consider $S_{0} \subset \bar{S}_{0}$ by setting $\psi(\phi)=\int_{0}^{1} \psi(t) \phi(t) d t$ for $\phi, \psi \in S_{0}$, and the conjugate modular $\bar{m}_{0}$ of $m_{0}$ is given by

$$
\bar{m}_{0}(\psi)=\int_{0}^{1} \frac{1}{\rho^{\prime}(t)}|\psi(t)|^{\rho^{\prime}(t)} d t \quad \text { for } \psi \in S_{0}
$$

if $1 / \rho(t)+1 / \rho^{\prime}(t)=1$ for $t \in[0,1]$, as proved in [1]. Thus, by (2) and (3), we can generalize the Hölder inequality as

$\int_{0}^{1}|\psi(t) \phi(t)| d t$

$$
\leq \operatorname{Max}_{r=p, q} r^{1 / r} r^{\prime^{1 / r^{\prime}}}\left(\int_{0}^{1} \frac{1}{\rho^{\prime}(t)}|\psi(t)|^{\rho^{\prime}(t)} d t\right)^{1 / r^{\prime}} \underset{r=p, q}{\operatorname{Max}}\left(\int_{0}^{1} \frac{1}{\rho(t)}|\phi(t)|^{\rho(t)} d t\right)^{1 / r}
$$

and

$$
\begin{aligned}
& \int_{0}^{1}|\psi(t) \phi(t)| d t \\
& \quad \leq \operatorname{Max}_{r=p, q}\left(\int_{0}^{1} \frac{1}{\rho^{\prime}(t)}|\psi(t)|^{\rho \prime(t)} d t\right)^{1 / r^{\prime}} \operatorname{Max}_{r=p, q} r^{1 / r} r^{\prime}{ }^{1 / r^{\prime}}\left(\int_{0}^{1} \frac{1}{\rho(t)}|\phi(t)|^{\rho(t)} d t\right)^{1 / r}
\end{aligned}
$$

if $1<p \leq \rho(t) \leq q$ and $1 / \rho(t)+1 / \rho^{\prime}(t)=1$ for $t \in[0,1], 1 / p+1 / p^{\prime}=1$, and $1 / q+1 / q^{\prime}=1$.

\section{REFERENCES}

1. H. Nakano, Topology and linear topological spaces, Maruzen, Tokyo, 1951; reprinted by University Microfilms, Ann Arbor, Mich., MR 13, 753.

2. - Generalized modular spaces, Studia Math. 31 (1968), 439-449. MR 38 \#2566.

3. $\longrightarrow, L_{p}$ modulars (to appear).

DEPARTMENT OF MATHEMATICS, WAYNE STATE UNIVERSITY, DETROIT, MICHIGAN 48202 